\section{Rates of retinal nerve fibre layer thickness change in glaucoma patients and control subjects}

\begin{abstract}
Purpose To examine the rates of retinal nerve fibre layer thickness (RNFLT) change in glaucoma patients and healthy, age-similar control subjects with three techniques: scanning laser polarimetry with variable corneal compensation (VCC) and enhanced corneal compensation (ECC), and timedomain optical coherence tomography (OCT). Methods Sixty-one patients and thirty-three controls were examined with each technique and with standard automated perimetry (SAP) every 6 months. Rates of global RNFLT change and SAP mean deviation (MD) change were estimated with linear mixedeffects models.
\end{abstract}

Results The median (interquartile range) baseline age was $64.4(58.2,71.0)$ years for patients and $62.4(56.3,70.1)$ years for controls $(P=0.56)$. There was a median of seven examinations over 3.1 years for patients and six examinations in $\mathbf{3 . 0}$ years for controls. Baseline visual field MD and RNFLT for all imaging modalities were significantly lower $(P<0.01)$ in patients compared with controls. Rates of RNFLT change were not significantly different between patients and controls $(P \geq 0.19)$. Mean rates of VCC-measured RNFLT change were -0.18 and $-0.37 \mu \mathrm{m}$ per year in patients and controls, whereas the respective figures for ECC and OCT were -0.13 and $-0.31 \mu \mathrm{m}$ per year, and 0.04 and $0.61 \mu \mathrm{m}$ per year. Mean rates of MD change were -0.20 and $0.03 \mathrm{~dB}$ per year in patients and controls, respectively $(P=0.01)$.

Conclusion Rates of RNFLT change in glaucoma patients were not statistically different from control subjects for any modality. A significantly negative rate of $\mathrm{MD}$ change in patients suggests a genuine, continued deterioration in these patients not reflected by RNFLT changes.
N O'Leary, PH Artes, DM Hutchison, MT Nicolela and $\mathrm{BC}$ Chauhan

Eye (2012) 26, 1554-1562; doi:10.1038/eye.2012.202; published online 19 October 2012

Keywords: retinal nerve fibre layer; glaucoma; progression; scanning laser polarimetry; optical coherence tomography

\section{Introduction}

Glaucoma leads to progressive loss of visual field sensitivity and to distinctive changes in the structure of the optic nerve head $(\mathrm{ONH})$ and retinal nerve fibre layer (RNFL). Rates of functional and structural deterioration in glaucoma can be highly variable between patients, ${ }^{1-4}$ however, it is important to detect rapid rates of change that potentially result in serious visual impairment, to enable appropriate intervention. Although standard automated perimetry (SAP) has been the most widely used method to assess glaucomatous progression, imaging of the $\mathrm{ONH}$ and RNFL also provides important measures by which anatomical changes associated with glaucoma can be measured. ${ }^{5-7}$ Both scanning laser polarimetry (SLP) and optical coherence tomography (OCT) imaging techniques offer quantitative and objective measures of RNFL thickness (RNFLT).

SLP is based on the principle of retardance of backscattered light passing through the presumed form birefringent RNFL, ${ }^{8,9}$ which can be translated to the thickness of the RNFL. The more recent versions of this technology, variable corneal compensation (VCC) ${ }^{10}$ and enhanced corneal compensation (ECC), ${ }^{11}$ rely on estimating the individual's corneal birefringence. ${ }^{12}$ Theoretically, the corneal birefringence can be better estimated and thus removed with ECC scanning, ${ }^{11}$ yielding a lower frequency of atypical birefringence pattern (ABP) measurements. ${ }^{13} \mathrm{ABP}$ is an artefact 
representing increased birefringence in the temporal and nasal quadrants not indicative of RNFLT patterns found with other methods or histology. OCT captures highresolution, three-dimensional images, with interferometry, of the retina and ONH from which RNFLT values can be derived. Time-domain OCT provides reproducible RNFLT measurements around the ONH. ${ }^{14}$ In this report, SLPVCC and SLP-ECC imaging modalities are referred to as VCC and ECC respectively, whereas time-domain OCT is referred to as OCT. Previous longitudinal studies have shown that VCC, ECC, and OCT can provide good discrimination in rates of RNFLT change between progressing and non-progressing glaucoma patients determined by visual field changes and/or expertassessed stereophotograph change. ${ }^{15-18}$

This was a longitudinal, prospective, observational study, the aims of which were to describe RNFLT changes over time in a cohort of glaucoma patients and agesimilar control subjects measured by VCC, ECC, and OCT. Rates of change in SAP mean deviation (MD) were also estimated and used to assess the differences in functional changes between glaucoma patients and control subjects.

\section{Materials and methods}

\section{Participants}

This study reports on data from patients with a clinical diagnosis of open-angle glaucoma, and healthy agesimilar control subjects, followed in a prospective longitudinal study. Patients were recruited consecutively from the glaucoma clinics of the Eye Care Centre at the Queen Elizabeth II Health Sciences Centre, whereas the control subjects were enroled from a local telephone company, seniors' groups, and local church organisations in Halifax, Nova Scotia. The study was approved by the Capital Health Research Ethics Committee and all participants gave written informed consent. All procedures conformed to the tenets of the Declaration of Helsinki.

For patients, inclusion criteria were: (i) clinical diagnosis of open-angle glaucoma, including primary, pseudoexfoliative or pigmentary glaucoma; (ii) a repeatable glaucoma hemifield test outside normal limits ${ }^{19}$ with SAP (Swedish Interactive Thresholding Algorithm (SITA) ${ }^{20}$ program 24-2 of the Humphrey Field Analyser (Carl Zeiss Meditec, Dublin, CA, USA)) with a maximum loss of $-12 \mathrm{~dB} \mathrm{MD}$; (iii) glaucomatous optic nerve head damage; and (iv) best-corrected visual acuity $\geq 0.3(20 / 40)$ logarithm minimum angle of resolution in the study eye. Exclusion criteria were: (i) concomitant ocular disease and systemic medication known to affect the visual field and (ii) refractive error exceeding $\pm 6.00 \mathrm{D}$ sphere or $\pm 3.00 \mathrm{D}$ astigmatism.
For healthy controls, inclusion criteria were: (i) normal eye examination with intraocular pressure $<21 \mathrm{~mm} \mathrm{Hg}$ and (ii) normal visual field defined as a glaucoma hemifield test, MD, and pattern SD within normal limits. The exclusion criterion was a refractive error exceeding $\pm 6.00 \mathrm{D}$ sphere or $\pm 3.00 \mathrm{D}$ astigmatism.

Both groups were followed identically with examinations every six months. At each examination, subjects had VCC, ECC, and OCT imaging and SAP (SITA 24-2) testing performed on one study eye. If both eyes were eligible, one eye was randomly selected as the study eye.

Subjects were imaged with a commercially available SLP device (GDx; Carl Zeiss Meditec), operating in both VCC and ECC modes (software version 5.4.1.35). The ECC mode was a non-commercial module. There were no functional differences between the ECC mode on this device and the commercial GDx-ECC. Subjects were also imaged with Stratus OCT (software version 5.1; Carl Zeiss Meditec). Imaging was performed by a single operator (DMH) throughout the study, and eyes were not dilated. In SLP imaging, the optic disc was delineated at each visit by the operator. RNFLT values were then calculated within an annular region, the inner circle of which could be of three different diameters: 2.4, 3.2, and $4.0 \mathrm{~mm}$ in emmetropic eyes with a constant width of $0.7 \mathrm{~mm}$ between inner and outer circles. This diameter was chosen to form a minimum-sized circle centred on the optic disc, which does not cross the optic disc boundary. RNFLT measurements, averaged across temporal-superior-nasal-inferior-temporal (TSNIT) sectors of this measurement annulus, are referred to as the TSNIT average. OCT imaging was performed with the commercially available OCT device (Stratus OCT; Carl Zeiss Meditec). The fast scan protocol was used during scanning, which acquired 256 equally spaced A-scans in a scanning circle, $3.4 \mathrm{~mm}$ in diameter, around the $\mathrm{ONH}$ three times in immediate sequence resulting in three sets of RNFLT measurements. RNFLT measurements calculated from each A-scan were averaged to form a global mean RNFLT value. The repeat function was used during follow-up scanning with the Stratus OCT to ensure the closest possible positioning of the scanning circle with those of previous scans. Calibration of each device (Stratus OCT and GDx) was performed regularly throughout the follow-up period and in accordance with the manufacturer's guidelines.

Intraocular surgery during follow-up was a selection factor for analysis of eyes. Previous studies have reported that cataract surgery results in an increase in measured RNFL retardance and thus SLP-measured RNFLT. ${ }^{21-23}$ Reports on the systematic differences of measured RNFLT pre- and post trabeculectomy are conflicting for OCT, ${ }^{24,25}$ and have been shown not to be significant for $\mathrm{SLP}^{26}$ In light of these findings, data from eyes with 
glaucoma surgery during follow-up were analysed, whereas those from eyes with cataract surgery were not.

Only eyes with $\geq 4$ examinations were included.

Figure 1 details the selection criteria and the data-set sizes at each level of selection. The scanning protocol of the study required two scans for each examination in the first 2 years of follow-up and one scan thereafter. Images were included if the following quality criteria were met. For VCC and ECC, images with a scan quality score (QS) $<7$ were excluded from analysis. The QS is used to summarise the illumination, intensity, centration on the $\mathrm{ONH}$, and contrast of the reflectance image of each scan. The typical scan score (TSS), developed to identify scans with an $\mathrm{ABP}$ caused by poor compensation for corneal birefringence, ${ }^{27}$ was not used as a means of excluding VCC or ECC images. OCT images were excluded if the commercial software issued a warning of either lowalgorithm confidence in segmenting the RNFL, insufficient signal strength (SS), or high noise by an internal software check, or if there was an obvious failure of the RNFL segmentation algorithm by visual inspection. All visual field tests had acceptable reliability indices: false-negative and false-positive rates of $<33 \%$; and fixation losses of $<20 \%$. Repeat scans were averaged to obtain one RNFLT measurement per examination. If scans from a given examination were excluded in one modality, the scans for other modalities and the SAP test, in the same examination, were also excluded.

\section{Statistical methods}

Linear mixed-effects models were used to estimate the rate of change of RNFLT during follow-up. Differences in the intercept and slope between groups were modelled as fixed effects, and the individual distribution of intercept and slope between subjects were modelled as random effects. Data were corrected for age, with baseline age modelled as a fixed effect. Changes in TSS leading to artefactual changes in RNFLT by VCC and ECC data were accounted for in the models. For a given measurement $j$ in subject $i$ at time $t_{i j}$ with measurement error $\varepsilon_{i j}$ the models of RNFLT and of visual field MD were specified as follows:

$$
\begin{aligned}
\operatorname{GDx}(\mathrm{RNFLT})_{i j}= & \beta_{0}+\beta_{1} D_{i}+\beta_{2} t_{i j}+\beta_{3} D_{i} t_{i j}+\beta_{4} A_{i} \\
& +\beta_{5} T_{i j}+b_{i 1}+b_{i 2} t_{i j}+\varepsilon_{i j} \\
\mathrm{OCT}(\mathrm{RNFLT})_{i j}= & \beta_{0}+\beta_{1} D_{i}+\beta_{2} t_{i j}+\beta_{3} D_{i} t_{i j}+\beta_{4} A_{i} \\
& +b_{i 1}+b_{i 2} t_{i j}+\varepsilon_{i j} \\
\operatorname{VF}(\mathrm{MD})_{i j}=\beta_{0}+ & \beta_{1} D_{i}+\beta_{2} t_{i j}+\beta_{3} D_{i} t_{i j}+\beta_{4} A_{i} \\
+ & b_{i 1}+b_{i 2} t_{i j}+\varepsilon_{i j}
\end{aligned}
$$

To investigate the differences between glaucoma and control subjects, the model incorporated a fixed effect for glaucoma $D_{i}$ and its interaction with time. The coefficient $\beta_{0}$ represents the mean baseline value of the control subjects, and $\beta_{0}+\beta_{1}$ represents the mean baseline of glaucoma patients. The rate of change in control subjects is given by $\beta_{2}$, such that $\beta_{2}+\beta_{3}$ gives the rate of change in the glaucoma patients. Random effects $b_{i 1}$ and $b_{i 2}$ represent the deviation of the intercept and rate of change in an individual subject from mean. Further corrections are made for baseline age $\left(A_{i}\right)$ and, in the VCC and ECC models of RNFLT (equation 1), for changes in TSS over time $\left(T_{i j}\right)$. The random effects are given by $b_{i 1}$, which represents the deviation of a subject's baseline measurement from the population average and $b_{i 2}$, which represents the deviation of a subject's rate of change from the population average. Rates of change in an individual subject $i$ can thus be obtained by calculating: $\beta_{2}+\beta_{3} D_{i}+b_{i 2}$.

The SD of residuals $\varepsilon_{i j}$ (SDRES) from the fitted model of each eye was used to estimate the variability in each series. The relationships between the mean image quality metric for a series obtained with a given modality (QS for VCC and ECC, and SS for OCT) and the SDRESs were quantified with Spearman's $\rho$.

For all hypothesis tests, statistical significance was set at a level of $P<0.05$. All analyses were performed using MATLAB (version R2009a; MathWorks, Natick, MA, USA) and R (version 2.15.0; http:/ / www.R-project.org/), using the linear and nonlinear mixed-effects model package. 28,29

\section{Results}

From an initial set of 70 glaucoma patients and 50 control subjects, the RNFLT series of 61 eyes of 61 glaucoma patients and 33 eyes of 33 control subjects were selected for analysis (Figure 1). A summary of the demographic variables and measurements at baseline of each group are shown in Table 1. Significant differences were observed between glaucoma patients and control subjects for baseline VCC, ECC, OCT RNFLT measurements and baseline visual field measurements. Groups were similar in age and gender, and in the length of follow-up and number of examinations. Because examinations were matched, each eye had the same number of measurements in the same follow-up period for all imaging modalities and SAP.

Table 2 shows the coefficients for each linear mixedeffects model. No statistically significant differences in rates of change were observed between glaucoma patients and control subjects for any RNFLT measurements (OCT: $P=0.32$, VCC: $P=0.19$, and ECC: $P=0.31)$. Significant differences $(P=0.01)$ were observed between the rates of MD change in glaucoma patients $(0.03 \mathrm{~dB}$ per year) and control subjects $(-0.20 \mathrm{~dB}$ per year). Distributions of rates of change of RNFLT 


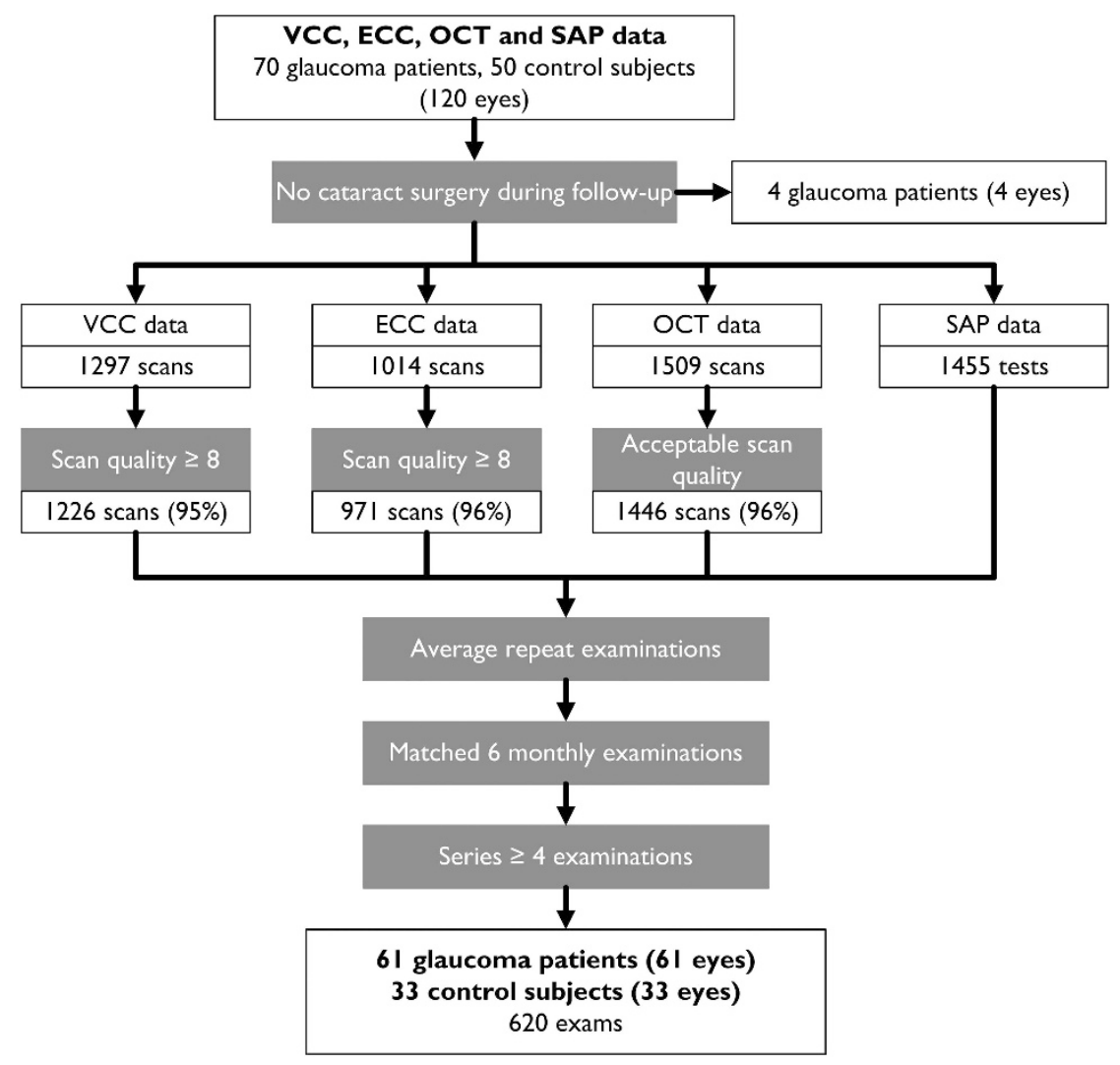

Figure 1 Study of selection criteria. Selection of data from VCC, ECC, and OCT imaging modalities, and SAP for analysis. Number of eyes in each group and total number of scans and examinations are shown.

measured by each modality for each group and mean rates of RNFLT change are displayed in Figure 2, along with distributions of rates of change of MD.

Median SDRES values were 1.1, 1.3 and $2.4 \mu \mathrm{m}$ for VCC-, ECC-, and OCT-measured RNFLT, respectively, for patients. Corresponding values were $0.9,0.9$, and $2.7 \mu \mathrm{m}$ for controls. SDRES values were weakly correlated with series-averaged QS for VCC RNFLT ( $\rho=-0.20, P=0.06)$, but this was not the case for ECC ( $\rho=-0.14, P=0.15)$. For OCT RNFLT, the SDRES values were not statistically significantly correlated with series-averaged SS ( $\rho=-0.11, P=0.27)$.

\section{Discussion}

This study reported longitudinal rates of change of RNFLT as measured by VCC, ECC, and OCT in a group of glaucoma patients and a group of age-similar control subjects. To the best of our knowledge, this is the first published study to report the rates of change for these three imaging modalities in concurrent data, and longitudinal rates of SLP- and OCT-measured RNFLT change in healthy control subjects. The quality and concurrency of the data analysed ensured reliability and consistency across modalities in follow-up.
Results showed no detectable increase in deterioration of global RNLFT in glaucoma patients compared with control subjects measured by either VCC or ECC imaging modalities. Rates derived from OCT were significantly more negative in glaucoma patients compared with control subjects. There was a significantly increased deterioration in SAP-measured MD in the glaucoma patients compared with control subjects, indicating that an overall functional deterioration did take place in glaucoma patients during the study. The rate measured in patients of $-0.20 \mathrm{~dB}$ per year was also consistent with reported rates of MD change in treated patients. ${ }^{30,31}$ Previous studies have reported mean rates from VCC, ECC, and OCT to be significantly more negative in progressing compared with non-progressing glaucoma patients. ${ }^{15-18,32,33}$ In these studies, progression was determined by visual field changes and/or expertassessed stereophotograph changes. However, this previous research is not directly comparable to the present study because glaucoma patients were not segregated into progressing or non-progressing categories. Because in many patients functional and structural change provides largely independent information about glaucoma progression, ${ }^{34-36}$ the use of 
visual field change as a reference standard to separate progressing and non-progressing patients to compare the merit of methods for measuring RNFLT change is problematic. Uncertainty with using independent reference standards is also compounded by the variability inherent in visual field measurements $\mathrm{s}^{37,38}$ and expert-assessed change in stereophotographs. ${ }^{39}$

Slow rates of RNFLT change in glaucoma patients could be accounted for by several factors. Because glaucoma patients were in a prospective study with regular and frequent examinations, it is possible that

Table 1 Demographic and baseline measurements, and followup details

\begin{tabular}{lccc}
\hline & Glaucoma & Control & $\mathrm{P}$ \\
\hline $\begin{array}{l}\text { Number of } \\
\text { subjects }\end{array}$ & 61 & 33 & \\
$\begin{array}{l}\text { Age at baseline } \\
\text { years })\end{array}$ & $64.4(58.2,71.0)$ & $62.4(56.3,70.1)$ & 0.56 \\
$\begin{array}{l}\text { Male } \\
\text { Female }\end{array}$ & $33(54 \%)$ & $12(36 \%)$ & $0.13^{*}$ \\
$\begin{array}{l}\text { TSNIT VCC } \\
\text { RNFLT }(\mu \mathrm{m})\end{array}$ & $43.4(38.1,47.6)$ & $52.3(49.0,55.7)$ & $<0.001$ \\
$\begin{array}{l}\text { TSNIT ECC } \\
\text { RNFLT }(\mu \mathrm{m})\end{array}$ & $38.8(35.6,43.8)$ & $49.5(46.9,52.5)$ & $<0.001$ \\
$\begin{array}{l}\text { Global OCT } \\
\text { RNFLT ( } \mu \mathrm{m})\end{array}$ & $69.1(59.8,80.5)$ & $98.7(93.2,103.9)$ & $<0.001$ \\
$\begin{array}{l}\text { Mean deviation } \\
\text { (dB) }\end{array}$ & $-3.6(-5.8,-0.1)$ & $0.0(-0.8,1.2)$ & $<0.001$ \\
$\begin{array}{l}\text { Pattern SD }(\mathrm{dB}) \\
\text { Follow-up }\end{array}$ & $4.1(1.9,9.8)$ & $1.6(1.4,1.9)$ & $<0.001$ \\
$\begin{array}{l}\text { length (years }) \\
\text { Number of } \\
\text { examinations }\end{array}$ & $3.1(2.8,3.5)$ & $3.0(2.6,3.2)$ & 0.06 \\
\hline
\end{tabular}

Abbreviations: ECC, enhanced corneal compensation; OCT, optical coherence tomography; RNFLT, retinal nerve fibre layer thickness; TSNIT, temporal-superior-nasal-inferior-temporal; VCC, variable corneal compensation.

Values shown are median (interquartile range); *Fisher's exact test, all others Mann-Whitney U-test. compliance to treatment was higher and possibly resulted in less progressive RNFLT loss. Thinner baseline RNFL in which further deterioration is less likely is another possible factor. The latter explanation is plausible for these patients as their median RNFLT at baseline was 43,39 and $69 \mu \mathrm{m}$ for VCC, ECC and OCT, respectively, values lower than previously reported. ${ }^{15-18,32,33}$ Despite this, these patients would be considered to have early to moderate glaucomatous damage by their visual field state (median MD: $-3.6 \mathrm{~dB}$ ) where changes are often considered to be more easily detected. Evidence is also emerging that in experimental glaucoma, changes in RNFL retardance ${ }^{40,41}$ and reflectance ${ }^{42}$ can precede changes in RNFLT, and this may explain a slower rate of SLP-measured RNFLT change in these patients. The large, but not statistically significant, difference between patients and controls in OCT-measured rates of RNFLT change was due in part to the substantial, positive rate of RNFLT change in control subjects. This observation has no obvious explanation and it is likely a chance finding as indicated by the lack of statistical significance. In contrast, a rate of RNFLT change with spectral-domain OCT in healthy control subjects of $-0.33 \mu \mathrm{m}$ per year has recently been reported. ${ }^{43}$ The lack of detectable differences between the rates of change of RNFLT in patients and controls can also be partially attributed to measurement variability. The medians of VCC, ECC, and OCT SDRES values in this study are similar to previous estimates of short- and long-term RNFLT variability measured by VCC, ECC, and OCT in glaucoma suspects and control subjects. ${ }^{44-46}$

In order to highlight how well these devices can detect clinically relevant RNFLT change over time, a simple model was derived. A linear decrease of RNFLT from the median baseline measurement in control subjects to that of glaucoma patients within 10 years was considered a meaningful rate of RNFLT change. These assumptions yielded rates of $-0.9,-1.1$, and $-3.0 \mu \mathrm{m}$ per year for

Table 2 Fixed-effects coefficients from linear mixed-effects model

\begin{tabular}{|c|c|c|c|c|c|c|c|c|}
\hline \multirow[t]{2}{*}{ Coefficient } & \multicolumn{2}{|c|}{ VCC RNFLT } & \multicolumn{2}{|c|}{ ECC RNFLT } & \multicolumn{2}{|c|}{ OCT RNFLT } & \multicolumn{2}{|l|}{$S A P M D$} \\
\hline & Estimate & $\mathrm{P}$ & Estimate & $\mathrm{P}$ & Estimate & $\mathrm{P}$ & Estimate & $P$ \\
\hline Rate of change in controls $\left(\beta_{3}\right)$ ( $\mu$ m per year) & -0.4 & 0.01 & -0.3 & 0.01 & 0.6 & 0.19 & $0.03(\mathrm{~dB}$ per year) & 0.68 \\
\hline Difference in rate of change $\left(\beta_{4}\right)$ ( $\mu$ m per year) & 0.2 & 0.31 & 0.2 & 0.19 & -0.6 & 0.32 & -0.23 (dB per year) & 0.01 \\
\hline Mean baseline in controls $\left(\beta_{1}\right)(\mu \mathrm{m})$ & 50.6 & $<0.001$ & 49.5 & $<0.001$ & 100.1 & $<0.001$ & $0.14(\mathrm{~dB})$ & 0.84 \\
\hline Difference in mean baseline $\left(\beta_{2}\right)(\mu \mathrm{m})$ & -10.3 & $<0.001$ & -10.3 & $<0.001$ & -31.1 & $<0.001$ & $-4.30(\mathrm{~dB})$ & $<0.001$ \\
\hline Baseline age $\left(\beta_{5}\right)$ ( $\mu$ m per year $)$ & -0.1 & 0.04 & -0.1 & 0.22 & -0.2 & 0.12 & -0.11 (dB per year) & $<0.01$ \\
\hline $\operatorname{TSS}\left(\beta_{6}\right)(\mu \mathrm{m} / \mathrm{U}$ score $)$ & -0.2 & $<0.001$ & -0.2 & $<0.001$ & $\mathrm{~N} / \mathrm{A}$ & $\mathrm{N} / \mathrm{A}$ & $\mathrm{N} / \mathrm{A}$ & $\mathrm{N} / \mathrm{A}$ \\
\hline
\end{tabular}

Abbreviations: ECC, enhanced corneal compensation; MD, mean deviation; OCT, optical coherence tomography; RNFLT, retinal nerve fibre layer thickness; SAP, standard automated perimetry; VCC, variable corneal compensation.

Values shown are fixed effects with coefficients from Equations 1-3 indicated. Rows in bold indicate the estimates of effects relating to the rate of change in control subjects $\left(\beta_{3}\right)$, and the difference between the rate of change in the controls and the glaucoma patients $\left(\beta_{4}\right)$. The mean rate of change in the glaucoma patients is given by $\beta_{3}+\beta_{4}$. 

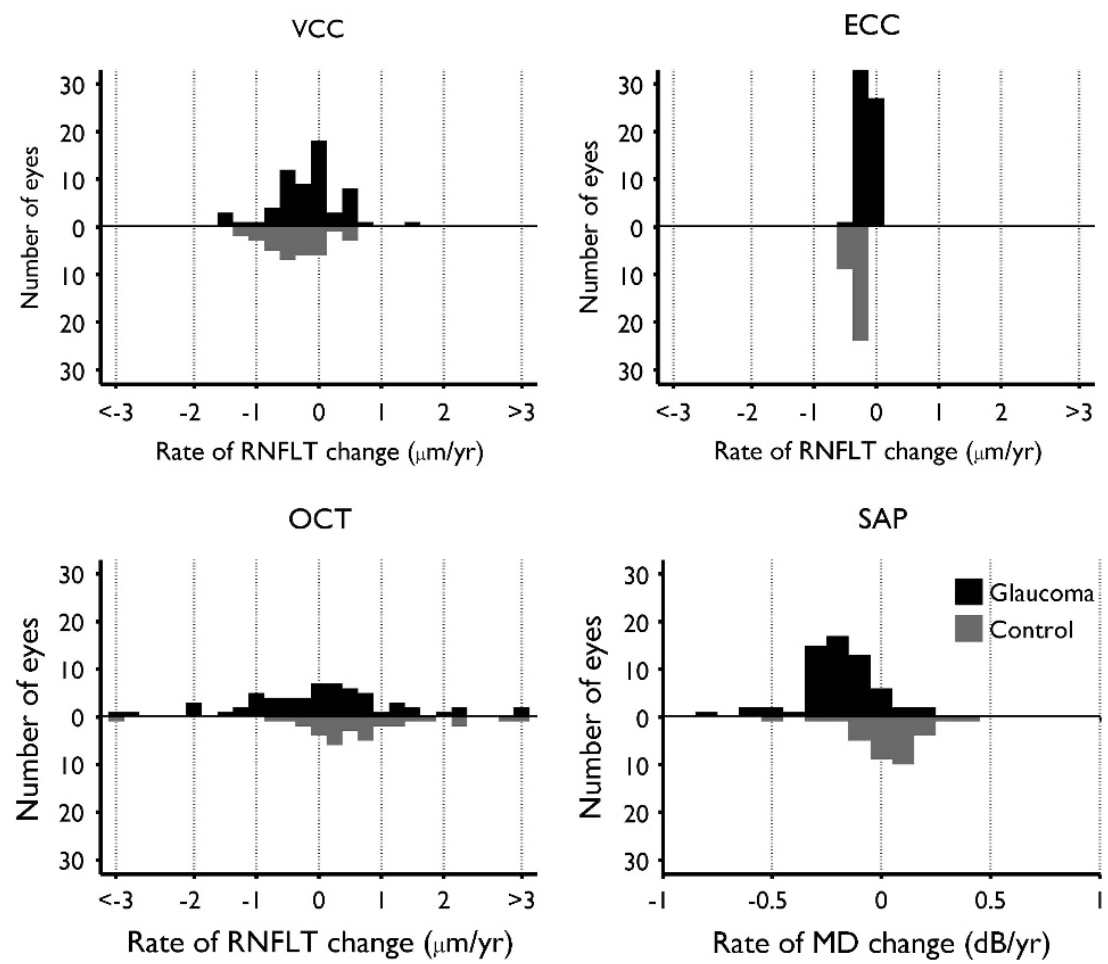

Figure 2 Distributions of RNFLT rates of change. Distributions of rates of globally averaged RNFLT change in the glaucoma patient (dark bars) and control subjects (light bars) for: VCC, ECC, and OCT. SAP MD rates for glaucoma patients and control subjects are also shown.

VCC, ECC, and OCT, respectively. Median and upperquartile SDRES estimates were used to model moderate and high levels of measurement variability in follow-up data. Measurements were modelled every 6 months over a follow-up period of 3.5 years (eight examinations), characteristic of the typical follow-up series in this study. The false-positive detection rate was fixed at $5 \%$. The resulting statistical power, in individuals, to detect RNFLT change was $0.74,0.79$, and 0.88 for VCC, ECC, and OCT, respectively, at moderate variability and 0.41 , 0.47 , and 0.56 for VCC, ECC, and OCT, respectively, at high variability. This indicates that all modalities have good power to detect clinically significant change at moderate variability.

Image quality metrics (QS for VCC and ECC and SS for OCT) were not strongly predictive of the variability of RNFLT measurements. This is largely consistent with previous research on the longitudinal reproducibility of VCC and OCT RNFLT. ${ }^{45}$ The birefringent properties of the cornea do not change significantly over time ${ }^{47}$ hence atypical retardance measurements also should not change. The TSS identifies atypical retardance patterns, ${ }^{27}$ hence changes in TSS may be predictive of spurious rates of RNFLT change. RNFLT was corrected for changes in TSS; this correction factor was found to be highly significant in both VCC and ECC with a consistent decrease of $0.2 \mu \mathrm{m}$ RNFLT per unit increase in TSS, in agreement with previous reports. ${ }^{48}$ Occurrence of atypical retardance patterns caused by anterior and posterior segment pathologies and, in particular, by disruption of the Henle fibre layer may be the cause of these changes; the expected birefringent behaviour of the Henle fibre layer is crucial in calibrating the compensation for corneal retardance. ${ }^{49}$

Alignment of serial scans may have a significant effect on the measured rates of RNFLT change. The new progression analysis with the latest commercial release of SLP provides alignment of follow-up images to baseline. This can be implemented for already acquired scans, with annular values recomputed for aligned images. Eye movements during image acquisition can produce measurement artefacts, typically leading to higher measured RNFLT. ${ }^{50}$ For the OCT, measurements are only obtained for the scanning circle and therefore postacquisition alignment cannot help to recalculate more reproducible RNFLT estimates. Image tracking on newer spectral-domain OCT devices minimises this source of error, ${ }^{51}$ and methods that use existing OCT data to measure the displacement of follow-up scans with respect to baseline can help interpret the estimated rates of RNFLT change. ${ }^{52}$

Measurement variability has an impact on the ability to detect underlying change and also the ability to resolve RNFLT change due to glaucoma from ageing. Through 
simple modelling, the power to detect a clinically meaningful rate of RNFLT change in individual patients with these devices was demonstrated to vary between approximately 40 and $90 \%$. These calculations were independent of any comparisons with external and potential confounding reference standards of glaucomatous progression.

VCC, ECC, and OCT may have a role in providing estimates of precise RNFLT changes, given that they provide measurements with good reproducibility. However, in this study, rates of RNFLT change from these modalities were not found to be different between glaucoma patients and normal controls, and the mean differences from zero change were small. In contrast, the rate of visual field deterioration in these patients was significantly greater compared with the control subjects, and the rate was similar to those reported previously. ${ }^{30,31}$ This makes it likely that the underlying changes in the RNFL of these glaucoma patients were small.

\section{Summary}

What was known before

- Changes in RNFL thickness have been reported separately for different imaging modalities, and differences have been shown between rates of RNFL thickness in groups of patients progressing by visual fields and those not.

- To our knowledge, rates of change of RNFL thickness in healthy control subjects have not yet been quantified with these devices.

What this study adds

- Rates of change in RNFL thickness over time in a group of moderately damaged glaucoma patients were, on average, small and not different to those of controls.

- This indicates that most RNFL thickness changes in patients may have occured in the earlier stages of disease before study enrolment, which accounts for baseline differences in RNFL between patients and controls.

\section{Conflict of interest}

The authors declare no conflict of interest.

\section{Acknowledgements}

This study was funded by the Grant MOP- 11357 from the Canadian Institute of Health Research (BCC). BCC has received research support from Carl Zeiss Meditec (Dublin, CA, USA).

\section{References}

1 Nicolela MT, McCormick TA, Drance SM, Ferrier SN, LeBlanc RP, Chauhan BC. Visual field and optic disc progression in patients with different types of optic disc damage: a longitudinal prospective study. Ophthalmology 2003; 110(11): 2178-2184.

2 Heijl A, Bengtsson B, Hyman L, Leske MC. Natural history of open-angle glaucoma. Ophthalmology 2009; 116(12): 2271-2276.

3 See JLS, Nicolela MT, Chauhan BC. Rates of neuroretinal rim and peripapillary atrophy area change: a comparative study of glaucoma patients and normal controls. Ophthalmology 2009; 116(5): 840-847.

4 Broman AT, Quigley HA, West SK, Katz J, Munoz B, Bandeen-Roche $\mathrm{K}$ et al. Estimating the rate of progressive visual field damage in those with open-angle glaucoma, from cross-sectional data. Invest Ophthalmol Vis Sci 2008; 49(1): 66-76.

5 Weinreb RN, Shakiba S, Zangwill L. Scanning laser polarimetry to measure the nerve fiber layer of normal and glaucomatous eyes. Am J Ophthalmol 1995; 119: 627-636.

6 Iester M, Broadway DC, Mikelberg FS, Drance SM. A comparison of healthy, ocular hypertensive, and glaucomatous optic disc topographic parameters. J Glaucoma 1997; 6(6): 363-370.

7 Bowd C, Weinreb RN, Williams JM, Zangwill LM. The retinal nerve fiber layer thickness in ocular hypertensive, normal, and glaucomatous eyes with optical coherence tomography. Arch Ophthalmol 2000; 118(1): 22-26.

8 Dreher AW, Reiter K. Scanning laser polarimetry of the retinal nerve fiber layer. SPIE 1992; 1746: 34-41.

9 Zhou Q, Knighton RW. Light scattering and form birefringence of parallel cylindrical arrays that represent cellular organelles of the retinal nerve fiber layer. Appl Opt 1997; 36(10): 2273-2285.

10 Zhou Q, Weinreb RN. Individualized compensation of anterior segment birefringence during scanning laser polarimetry. Invest Ophthalmol Vis Sci 2002; 43(7): 2221-2228.

11 Zhou Q. Retinal scanning laser polarimetry and methods to compensate for corneal birefringence. Bull Soc Belge Ophtalmol 2006; 302: 89-106.

12 Greenfield DS, Knighton RW, Huang XR. Effect of corneal polarization axis on assessment of retinal nerve fiber layer thickness by scanning laser polarimetry. Am J Ophthalmol 2000; 129(6): 715-722.

13 Morishita S, Tanabe T, Yu S, Hangai M, Ojima T, Aikawa H et al. Retinal nerve fibre layer assessment in myopic glaucomatous eyes: comparison of GDx variable corneal compensation with GDx enhanced corneal compensation. Br J Ophthalmol 2008; 92(10): 1377-1381.

14 Budenz DL, Chang RT, Huang X, Knighton RW, Tielsch JM. Reproducibility of retinal nerve fiber thickness measurements using the stratus OCT in normal and glaucomatous eyes. Invest Ophthalmol Vis Sci 2005; 46(7): 2440-2443.

15 Medeiros FA, Alencar LM, Zangwill LM, Bowd C, Vizzeri $\mathrm{G}$, Sample PA et al. Detection of progressive retinal nerve fiber layer loss in glaucoma using scanning laser polarimetry with variable corneal compensation. Invest Ophthalmol Vis Sci 2009; 50(4): 1675-1681.

16 Alencar LM, Zangwill LM, Weinreb RN, Bowd C, Sample PA, Girkin CA et al. A comparison of rates of change in neuroretinal rim area and retinal nerve fiber layer thickness in progressive glaucoma. Invest Ophthalmol Vis Sci 2010; 51(7): 3531-3539.

17 Medeiros FA, Zangwill LM, Alencar LM, Sample PA, Weinreb RN. Rates of progressive retinal nerve fiber layer loss in glaucoma measured by scanning laser polarimetry. Am J Ophthalmol 2010; 149(6): 908-915. 
18 Medeiros FA, Zangwill LM, Alencar LM, Bowd C, Sample PA, Susanna Jr R et al. Detection of glaucoma progression with Stratus OCT retinal nerve fiber layer, optic nerve head, and macular thickness measurements. Invest Ophthalmol Vis Sci 2009; 50(12): 5741-5748.

19 Asman P, Heijl A. Glaucoma hemifield test. automated visual field evaluation. Arch Ophthalmol 1992; 110: 812-819.

20 Bengtsson B, Olsson J, Heijl A, Rootzen H. A new generation of algorithms for computerized threshold perimetry, SITA. Acta Ophthalmol Scand 1997; 75(4): 368-375.

21 Kremmer S, Garway-Heath DF, De Cilla S, Steuhl KP, Selbach JM. Influence of cataract surgery with implantation of different intraocular lenses on scanning laser tomography and polarimetry. Am J Ophthalmol 2003; 136(6): 1016-1021.

22 Gazzard G, Foster PJ, Devereux JG, Oen F, Chew PT, Khaw PT et al. Effect of cataract extraction and intraocular lens implantation on nerve fibre layer thickness measurements by scanning laser polarimeter (GDx) in glaucoma patients. Eye (Lond) 2004; 18(2): 163-168.

23 Dada T, Behera G, Agarwal A, Kumar S, Sihota R, Panda A. Effect of cataract surgery on retinal nerve fiber layer thickness parameters using scanning laser polarimetry (GDx VCC). Indian J Ophthalmol 2010; 58(5): 389-394.

24 Chang PT, Sekhon N, Budenz DL, Feuer WJ, Park PW, Anderson DR. Effect of lowering intraocular pressure on optical coherence tomography measurement of peripapillary retinal nerve fiber layer thickness. Ophthalmology 2007; 114(12): 2252-2258.

25 Aydin A, Wollstein G, Price LL, Fujimoto JG, Schuman JS. Optical coherence tomography assessment of retinal nerve fiber layer thickness changes after glaucoma surgery. Ophthalmology 2003; 110(8): 1506-1511.

26 Yamada N, Chen PP, Mills RP, Leen MM, Stamper RL, Lieberman MF et al. Glaucoma screening using the scanning laser polarimeter. J Glaucoma 2000; 9(3): 254-261.

27 Bagga H, Greenfield DS, Feuer WJ. Quantitative assessment of atypical birefringence images using scanning laser polarimetry with variable corneal compensation. Am J Ophthalmol 2005; 139(3): 437-446.

28 Pinheiro J, Bates D, DebRoy S, Sarkar D. Team RDC. NLME: linear and nonlinear mixed effects models. $R$ package 2012; version 3.1-104.

29 Pinheiro JC, Bates DM. Mixed-effects models in S and S-PLUS Springer: New York, 2000.

30 Smith SD, Katz J, Quigley HA. Analysis of progressive change in automated visual fields in glaucoma. Invest Ophthalmol Vis Sci 1996; 37: 1419-1428.

31 Chauhan BC, Mikelberg FS, Artes PH, Balazsi AG, LeBlanc RP, Lesk MR et al. Canadian glaucoma study: 3. Impact of risk factors and intraocular pressure reduction on the rates of visual field change. Arch Ophthalmol 2010; 128(10): 1249-1255.

32 Leung CK, Cheung CYL, Weinreb RN, Qiu K, Liu S, Li H et al. Evaluation of retinal nerve fiber layer progression in glaucoma: a study on optical coherence tomography guided progression analysis. Invest Ophthalmol Vis Sci 2010; 51(1): 217-222.

33 Wollstein G, Schuman JS, Price LL, Aydin A, Stark PC, Hertzmark E et al. Optical coherence tomography longitudinal evaluation of retinal nerve fiber layer thickness in glaucoma. Arch Ophthalmol 2005; 123(4): 464-470.
34 Artes PH, Chauhan BC. Longitudinal changes in the visual field and optic disc in glaucoma. Prog Retin Eye Res 2005; 24(3): 333-354

35 Strouthidis NG, Scott A, Peter NM, Garway-Heath DF. Optic disc and visual field progression in ocular hypertensive subjects: detection rates, specificity, and agreement. Invest Ophthalmol Vis Sci 2006; 47(7): 2904-2910.

36 Hudson CJW, Kim LS, Hancock SA, Cunliffe IA, Wild JM. Some dissociating factors in the analysis of structural and functional progressive damage in open-angle glaucoma. $\mathrm{Br} \mathrm{J}$ Ophthalmol 2007; 91(5): 624-628.

37 Wild JM, Pacey IE, Hancock SA, Cunliffe IA. Betweenalgorithm, between-individual differences in normal perimetric sensitivity: full threshold, FASTPAC, and SITA. Swedish Interactive Threshold algorithm. Invest Ophthalmol Vis Sci 1999; 40(6): 1152-1161.

38 Artes PH, Iwase A, Ohno Y, Kitazawa Y, Chauhan BC. Properties of perimetric threshold estimates from full threshold, SITA standard, and SITA fast strategies. Invest Ophthalmol Vis Sci 2002; 43(8): 2654-2659.

39 Jampel HD, Friedman D, Quigley H, Vitale S, Miller R, Knezevich F et al. Agreement among glaucoma specialists in assessing progressive disc changes from photographs in open-angle glaucoma patients. Am J Ophthalmol 2009; 147(1): 39-44; e31.

40 Fortune B, Cull GA, Burgoyne CF. Relative course of retinal nerve fiber layer birefringence and thickness and retinal function changes after optic nerve transection. Invest Ophthalmol Vis Sci 2008; 49(10): 4444-4452.

41 Fortune B, Burgoyne CF, Cull GA, Reynaud J, Wang L. Structural and functional abnormalities of retinal ganglion cells measured in vivo at the onset of optic nerve head surface change in experimental glaucoma. Invest Ophthalmol Vis Sci 2012; 53(7): 3939-3950.

42 Huang X-R, Zhou Y, Kong W, Knighton RW. Reflectance decreases before thickness changes in the retinal nerve fiber layer in glaucomatous retinas. Invest Ophthalmol Vis Sci 2011; 52(9): 6737-6742.

43 Leung CK, Yu M, Weinreb RN, Ye C, Liu S, Lai G et al. Retinal nerve fiber layer imaging with spectral-domain optical coherence tomography: a prospective analysis of age-related loss. Ophthalmology 2012; 119(4): 731-737.

44 Medeiros FA, Doshi R, Zangwill LM, Vasile C, Weinreb RN. Long-term variability of GDx VCC retinal nerve fiber layer thickness measurements. J Glaucoma 2007; 16(3): 277-281.

45 Leung CK, Cheung CY, Lin D, Pang CP, Lam DS, Weinreb $\mathrm{RN}$. Longitudinal variability of optic disc and retinal nerve fiber layer measurements. Invest Ophthalmol Vis Sci 2008; 49(11): 4886-4892.

46 Mai TA, Reus NJ, Lemij HG. Retinal nerve fiber layer measurement repeatability in scanning laser polarimetry with enhanced corneal compensation. J Glaucoma 2008; 17(4): 269-274

47 Mai TA, Lemij HG. Longitudinal measurement variability of corneal birefringence and retinal nerve fiber layer thickness in scanning laser polarimetry with variable corneal compensation. Arch Ophthalmol 2008; 126(10): 1359-1364.

48 Medeiros FA, Alencar LM, Zangwill LM, Sample PA, Susanna Jr R, Weinreb RN. Impact of atypical retardation patterns on detection of glaucoma progression using the GDx with variable corneal compensation. Am J Ophthalmol 2009; 148(1): 155-163, e151. 
49 Knighton RW, Huang XR, Greenfield DS. Analytical model of scanning laser polarimetry for retinal nerve fiber layer assessment. Invest Ophthalmol Vis Sci 2002; 43(2): 383-392.

50 Colen TP, Lemij HG. Motion artifacts in scanning laser polarimetry. Ophthalmology 2002; 109(8): 1568-1572.

51 Langenegger SJ, Funk J, Toteberg-Harms M. Reproducibility of retinal nerve fiber layer thickness measurements using the eye tracker and the retest function of Spectralis SD-OCT in glaucomatous and healthy control eyes. Invest Ophthalmol Vis Sci 2011; 52(6): 3338-3344.

52 Zhu H, Crabb D, Schlottmann P, Wollstein G, GarwayHeath D. Aligning scan acquisition circles in optical coherence tomography images of the retinal nerve fibre layer. IEEE Trans Med Imaging 2011; 30(6): 1228-1238. 